\title{
Summary of the Yield of Endometrial Aspiration in Women with Various Risk Factors and Bleeding Abnormalities
}

\author{
Roya Tabatabai ${ }^{1 *}$, Anita L Nelson ${ }^{2}$ and Lisa Vasquez ${ }^{2}$ \\ ${ }^{1}$ Olive View Medical Center, USA \\ ${ }^{2}$ Harbor-UCLA Medical Center, USA \\ *Corresponding author: Roya Tabatabai, Olive View Medical Center, USA, Tel: +818-648-6158, E-mail: rtabatabai@gmail.com
}

Received date: January 26, 2017; Accepted date: April 11, 2017; Published date: April 17, 2017

Citation: Tabatabai R, Nelson AL, Vasquez L (2017) Summary of the yield of endometrial aspiration in women with various risk factors and bleeding abnormalities. Reproductive Immunol Open Acc 2: 30. doi: 10.21767/2476-1974.100030

Copyright: (C) 2017 Tabatabai R, et al. This is an open-access article distributed under the terms of the Creative Commons Attribution License, which permits unrestricted use, distribution, and reproduction in any medium, provided the original author and source are credited.

\section{Rapid Communication}

Abnormal uterine bleeding (AUB), defined as prolonged, heavy, intermenstrual or postmenopausal bleeding, is a common presenting complaint for women seeking gynecologic consult. Endometrial carcinoma, which is the most frequent gynecologic malignancy in the United States, often presents with abnormal uterine bleeding. As a result, endometrial sampling is part of the diagnostic evaluation for AUB in women with risk factors, including obesity, null parity, significant family history and postmenopausal bleeding [1-3]. The specific indication for performing endometrial biopsy, however, varies amongst expert panels. For instance, the Canadian Society of Obstetricians and Gynecologists recommends endometrial sampling women over 40 years of age with AUB [4] whereas the American Congress of Obstetricians and Gynecologists recommends testing in women as young as 19 years of age who do not respond to medical therapy or with prolonged periods of unopposed estrogen stimulation [5]. With the understanding that AUB is more likely to be caused by benign rather than malignant pathology and given lack of consensus recommendations on the topic, we sought to determine the yield of endometrial sampling amongst women in various risk categories. Diagnostic yield is an important piece of information to allow patients and providers accurately discuss risks versus benefits of invasive procedures.

To determine diagnostic yield, we performed a retrospective chart review of women presenting to Harbor-UCLA Medical Center in Torrance, California between 2001 and 2008 who underwent endometrial biopsy to evaluate abnormal bleeding. Data abstracted from the charts included age, comorbid medical conditions, body mass index (BMI), pattern of abnormal bleeding, and histologic results of the biopsy specimen. We defined significant abnormal pathology as either carcinoma or hyperplasia with atypia, which is a pre-malignant condition. Yield was expressed as the percentage of samples with either atypia or carcinoma and Fisher's two-tailed exact test was used to compare frequencies of outcomes.

We reviewed charts of 1601 women in this study. Most of the women were obese as defined by $\mathrm{BMI}>30(58 \%)$ with a mean BMI of 33; nearly half of women (48.3\%) were peri-menopausal (age 41-50). In terms of comorbid conditions, nearly $15 \%$ volunteered a diagnosis of diabetes mellitus. Below are highlights of the results of our study.

- Of the 1601 women, a total of 101 were found to have a diagnosis of carcinoma or atypia and as expected, the yield of a positive result differed amongst subgroups.

- The incidence of carcinoma did not differ significantly between women with a $\mathrm{BMI}<30$ vs. BMI>30, however atypical hyperplasia was significantly more frequent among those with $\mathrm{BMI}>30$ (4.0\% vs. $4.9 \%, \mathrm{p}=0.02)$.

- After stratifying patients according to age groups $\quad<40$, $41-50,>50)$, yield of atypia was increased in women with $\mathrm{BMI}>30$ as compared to those with lower $\mathrm{BMI}$ in each age group. The impact of BMI was greatest in the youngest age category.

- In each age group, women with diabetes had higher rates of carcinoma or atypia.

- When examining yield of pathology according to pattern of abnormal bleeding, postmenopausal bleeding was associated with the highest yield of significant disease of (16.7\%), whereas among women with normal menstrual intervals but prolonged or excessive bleeding only a $1.3 \%$ had a chance of atypia or carcinoma.

- The overall highest yield (22.4\%) was seen in postmenopausal women with $\mathrm{BMI}>30$ and diabetes 
- The following Table 1 summarizes the above findings. The table shows percentage of patients with significant abnormal pathology stratified by age, BMI, and presence or absence of diabetes.

Table 1: BMI body mass index in $\mathrm{kg} / \mathrm{m}^{2}$, DM diabetes mellitus; ${ }^{*}$ no patients sampled.

\begin{tabular}{|c|c|c|c|c|c|c|c|c|c|c|c|c|}
\hline & \multicolumn{4}{|c|}{ Age $<40$ years } & \multicolumn{4}{|c|}{ Age $41-50$ years } & \multicolumn{4}{|c|}{ Age $>50$ years } \\
\hline & \multicolumn{2}{|l|}{$\mathrm{BMI}<30$} & \multicolumn{2}{|c|}{$\mathrm{BMI}>30$} & \multicolumn{2}{|l|}{$\mathrm{BMI}<30$} & \multicolumn{2}{|l|}{$\mathrm{BMI}>30$} & \multicolumn{2}{|c|}{$\mathrm{BMI}<30$} & \multicolumn{2}{|c|}{$\mathrm{BMI}>30$} \\
\hline & No DM & DM & $\begin{array}{l}\text { No } \\
\text { DM }\end{array}$ & DM & No DM & DM & No DM & $\mathrm{DM}$ & $\begin{array}{l}\text { No } \\
\text { DM }\end{array}$ & DM & $\begin{array}{l}\text { No } \\
\text { DM }\end{array}$ & DM \\
\hline Number of Subjects & 132 & 11 & 226 & 30 & 334 & 23 & 347 & 73 & 146 & 24 & 179 & 76 \\
\hline $\begin{array}{ll}\% \text { of patients } & \text { with } \\
\text { hyperplasia } & \text { with } \\
\text { atypia } & \end{array}$ & 0.8 & 0 & 2.2 & 6.7 & 0.8 & * & 2.3 & 4.1 & 0.7 & * & 0.6 & 3.9 \\
\hline $\begin{array}{l}\% \text { of patients with } \\
\text { carcinoma }\end{array}$ & 1.5 & 0 & 2.7 & 6.7 & 1.2 & * & 0.9 & 4.1 & 11.6 & 16.7 & 8.4 & 22.4 \\
\hline
\end{tabular}

The question our study sought to answer was what is the likelihood that a woman with AUB with a given age and BMI would have significant pathology on endometrial sampling. In our retrospective review, we noted that yield differed according to age, pattern of abnormal bleeding and BMI. We hope that this information can help both patients and providers make the best informed decisions by integrating diagnostic yield into the risk/benefit equation for endometrial sampling. In a subset of patients with demonstrated low yield of abnormal pathology such as young women with normal menstrual intervals but excess or prolonged bleeding, it may be reasonable to defer endometrial sampling and employ empiric treatment with progestin as a first step in management of their AUB. We hope that this data contribute to research in this area to help better define indications for performing endometrial biopsy $[4,5]$.

Our study population had a higher prevalence of risk factors and of endometrial carcinoma and atypical carcinoma than has been reported in previous studies, allowing us to provide a more secure estimate of the upper limit of the potential for disease detected by endometrial aspiration. Inherent to limitations of retrospective chart reviews, data may not have been as accurate as what would be provided by prospective data, and some information in individual patient records was incomplete due to insufficient charting.

\section{References}

1. Nelson AL, Vasquez L, Tabatabai R, IM SS (2016) The yield of endometrial aspiration in women with various risk factors and bleeding abnormalities. Contracep Repro Med 1: 9.

2. Berek JS, Novak E (2007) Berek \& Novak's Gynecology: Lippincott Williams \& Wilkins.

3. Novak E, Berek JS (2002) Novak's Gynecology: Lippincott Williams \& Wilkins.

4. Vilos GA, Lefebvre G, Graves GR (2001) Guidelines for the management of abnormal uterine bleeding. J Obstet Gynaecol Can 23: 704-709.

5. Committee on Practice Bulletins (2013) Gynecology. Practice Bulletin No. 136: Management of abnormal uterine bleeding associated with ovulatory dysfunction. Obstet Gynecol 122: 176-185. 